Jakob Michael Perschy*

\title{
Die Burgenländische Landesbibliothek - ein offenes Fenster nach Osten
}

https://doi.org/10.1515/bfp-2018-0055

Zusammenfassung: Das heutige Burgenland wurde nach dem I. Weltkrieg aufgrund des Vertrages von Trianon 1921 von Ungarn an Österreich abgetreten und bildet seit damals ein Bundesland der Republik Österreich. Die „Burgenländische Landesbibliothek“ konnte bei ihrer Gründung im Jahre 1922 gerade einmal auf einem Grundstock in Form einer Dublettenspende von knapp 3000 Bänden der Österreichischen Nationalbibliothek aufbauen. In ihren ersten Jahren hauptsächlich als eine Art Handbibliothek für Regierung und Verwaltung genützt, wandelte sie sich bald in eine landeskundliche Forschungsbibliothek mit starkem historischen Schwerpunkt. Dies brachte mit sich, dass eine große Zahl an Schriftgut aus dem vormaligen Königreich Ungarn in die Bestände einfloss; ein geschichtsbedingter Umstand, dem auch heute noch, bei einem Gesamtbestand von 140000 Bänden, Rechnung getragen wird, was diese Bibliothek zu einem weit offenen Fenster nach Osten macht.

Schlüsselwörter: Burgenland; Bibliothek; Geschichte; Ungarn; Osteuropa; Zeitgeschichte

\section{An Austrian Library Considering Hungarian History}

Abstract: Burgenland is the only area of Austria, which was belonging to the Kingdom of Hungary before World War I. Founded in 1922, the Library of Burgenland was extended upon a base of less than 3,000 tomes, gifted by the National Library of Austria. As a matter of his history, it began to collect different kinds of literature and records from and about the historical Hungary. This collection strategy was maintained and expanded to contemporary history, what makes this library looks like a wide open window to the European East.

Keywords: Burgenland; library; history; Hungary; Eastern Europe; contemporary history

*Kontaktperson: Dr. Jakob Michael Perschy, Jakob.Perschy@bgld.gv.at
Es handelte sich um ein ausgesprochen rurales Gebiet, das da, drei Jahre nach dem Ende der Habsburgermonarchie, von deren ehemals ungarischen Reichshälfte zu der von der österreichischen Reichshälfte verblieben Republik wechselte. Eine Region ohne ein städtisches Zentrum, fast ohne Bildungseinrichtungen, ein agrarisch strukturiertes Land der Dörfer, welches seit dem späten Jahr 1921 unter der Bezeichnung „Burgenland“ als neues Bundesland der Republik Österreich firmierte. Auf den Namen war man verfallen, weil es sich bei dem schmalen, sich aber weit von Norden nach Süden hinziehenden Landstrich um die westlichen Teile dreier ungarischer Komitate handelte, welche in deutscher Sprache Wieselburg, Ödenburg und Eisenburg genannt wurden, als dreimal „Burg“, warum dann nicht „Burgenland“. Die Frage einer Hauptstadt bzw. eines Regierungssitzes des neuen Bundeslandes galt es damals, Ende 1921, noch zu lösen.

Die Stadt Sopron/Ödenburg war ja so etwas wie ein natürlich gewachsenes urbanes Zentrum der Region und deren nationaler Wechsel nach Österreich eingeplant gewesen, allerdings beharrte Ungarn auf einer Volksabstimmung, welche schließlich auch zu dessen Gunsten entschieden wurde. Im Landes selbst existierten nun einige größere Marktflecken, die für ihre jeweiligen Umgebungen zentrale Funktionen und ansatzweise städtische Strukturen entwickelt hatten, sowie zwei sehr hübsche barocke Kleinststädtchen, ehemals königlich-ungarische Freistädte, die ihren Status dem florierenden Weinbau und -handel der frühen Neuzeit verdankten, jedoch in ihrem Wesen weitgehend Ackerbürgerstädtchen geblieben waren. Angedacht wurde unter anderem, die Verwaltung des Burgenlandes im niederösterreichischen Wiener Neustadt zu installieren, zumal dieser Stadt eine bezüglich der Bevölkerung des Burgenlandes geografisch eine zumindest ziemlich zentrale Lage zukam.

Da man jedoch dem neuen Bundesland keinen extraterritorialen Regierungssitz antun wollte, wählte man vorerst doch Bad Sauerbrunn, einen kleinen, einstmals feinen Kurort mit einigen schönen Jugendstilvillen, der vor allem von Wien aus relativ rasch zu erreichen war. In einer der erwähnten Villen, die von der Regierung ihren Besitzern abgelöst wurden, nämlich in der „Tannenblatt-Villa“, entstand im Jahre 1922 die Burgenländische Landesbibliothek, auf Grundlage einer 2992 Bände umfassenden Du- 
blettenspende seitens der Österreichischen Nationalbibliothek. In ihren ersten Jahren konnte unsere Landesbibliothek auch kaum eine andere Rolle, als die einer - in erster Linie juridischen - Handbibliothek von Verwaltung und Regierung, einnehmen. Das änderte sich erst mit der Lösung der Hauptstadtfrage im Jahre 1925, als Eisenstadt das größere und verkehrstechnisch günstiger gelegene der beiden erwähnten Barockstädtchen - zum Regierungssitz des Burgenlandes erkoren wurde.

Zusammen mit dem Landesarchiv und dem Landesmuseum bezog die Landesbibliothek ein eigenes Gebäude in der neuen Hauptstadt Eisenstadt, das sogenannte „Leinner-Haus“, das zunächst auch das Landesmuseum beherbergte, und konnte sich damit von da an vermehrt, wenn auch mit bescheidenen Mitteln, als Fachbibliothek der landeskundlichen Forschung etablieren. Zu jener Zeit war die Genese des Burgenlandes bei weitem noch nicht abgeschlossen, die Verkehrsverbindungen zwischen dem Norden und dem Süden des Landes mehr als sehr verbesserungswürdig, was auch auf ein sogenanntes „Landesbewusstsein“ zutreffend gewesen sein mag. Die Identitätsfindung des Burgenlandes beschränkte sich zunächst auf eine strikte Ablehnung alles „Magyarischen“ - womit man wohl die aus mehreren Gründen nicht gänzlich unantastbare Rechtmäßigkeit der Abtretung des Gebietes an Österreich zumindest in den Köpfen der Bevölkerung legitimiert haben wollte - sowie einer Betonung des „deutschen Charakters" unseres Landstriches, wobei sowohl Volkskundler als auch Sprachforscher ordentlich ihre Phantasie in Anspruch nahmen. Damit Hand in Hand ging jedoch eine historisch gedachte - Solidarisierung mit jenen deutschsprachigen Bevölkerungsgruppen, die nach wie vor in Ländern mit anderssprachiger Bevölkerungsmehrheit in Ostmittel- und Südosteuropa lebten.

Die historisch-landeskundliche Forschung im Burgenland verweigerte zumindest zum Teil den oben erwähnten Antimagyarismus, hatte jedoch gegen vergleichende Forschungen bezüglich anderer deutschsprachiger Gebiete des alten Königreichs Ungarn nichts einzuwenden. In praxi bedeutete dies für die Landesbibliothek, dass nicht nur historische Werke unseres westungarisch/burgenländischen Raumes angeschafft wurden, sondern auch schon zur damaligen Zeit - hauptsächlich, jedoch nicht ausschließlich deutschsprachige - Literaturen aus allen Teilen des ehemaligen Königreichs Ungarn gesammelt wurden. Somit war unserer Landesbibliothek großes Fenster nach dem Osten (fast) von Anfang an weit geöffnet; ein Umstand, der uns bis heute von allen anderen österreichischen Bibliotheken zu unterscheiden vermag.

Durch die Herausgabe der heute noch existierenden „Burgenländischen Heimatblätter“ seit 1927 (bis 1932 noch unter dem Titel „Burgenländische Vierteljahrshefte“) schufen Landesarchiv und -bibliothek gemeinsam ein Organ zur Landeskunde, welches heute als eines der wichtigsten Quellenwerke der historischen Forschung im Burgenland gelten kann. Die Entwicklung des Bibliotheksbestandes hielt sich indes vor allem aufgrund der prekären finanziellen Situation dieser Zwischenkriegsjahre in relativ engen Grenzen; man kann von einem Zuwachs von nicht wesentlich mehr als 500 Bänden per anno ausgehen. Ein hoher Prozentsatz der damaligen Neuerwerbungen dürfte in Ungarn getätigt worden sein. Es handelt sich dabei hauptsächlich um deutschsprachige oder ins Deutsche übertragene Monografien zur Geschichte Ungarns, welche zur historischen Abhandlung des nun österreichischen Bundeslandes allerdings unumgänglich waren.

Die große Zäsur von 1938, der nicht rechtens gewesene aber doch gebilligte und einzig mexikanischen Widerspruch auslösende Anschluss Österreichs an das Deutsche Reich, bedeutete für das immer noch junge Bundesland kaum 17 Jahre war es alt geworden - dessen Auflösung, den neuen Machthabern erschien ein eigener „Reichsgau Burgenland“ zu wenig und gering, es war ja nun „klotzen statt kleckern" angesagt, da half auch nichts, dass burgenländische Nationalsozialisten in den Jahren zuvor schon brave braune Wühlarbeit geleistet hatten. Somit fiel der Norden des Burgenlandes an den „Reichsgau Niederdonau“, sein Süden an den „Reichsgau Steiermark“ und die Burgenländische Landesbibliothek wurde stillgelegt, eine Maßnahme, welche die betroffene Bevölkerung wahrscheinlich am wenigsten hart getroffen haben wird. Die Bestände unserer Bibliothek fanden sich jedenfalls in Kisten verpackt und ins „Landhaus“, dem Sitz der vormaligen Landesregierung verfrachtet, als dieses am letzten Märztag des Jahres 1945 von abziehenden Gestapoleuten in Brand gesteckt wurde. Denen ging es zwar mehr um die sie belastenden Akten und weniger um Monografien zur Geschichte des Königreichs Ungarn, trotzdem fiel rund ein Viertel des Bibliotheksbestandes den Flammen zum Opfer; höchst bedauerlicher Weise auch sämtliche Eingangsbücher der Bibliothek (weshalb ich auch meine obigen Angaben zur Bestandsentwicklung der Zwischenkriegszeit im Konjunktiv machen musste).

Im Oktober 1945 wurden das Bundesland Burgenland und auch dessen Landesbibliothek reinstalliert. Zehntausend Bände zählte die wiedererrichtete Bibliothek und war damit noch wirklich bescheidenen Zuschnitts. Nach Überwindung der Trümmer- und Mangeljahre (Zuwachs 1946: 131 Bände) wurde eine umfassendere Erwerbspolitik eingeleitet, welche auch die vordem etwas stiefmütterlich behandelte burgenländisch-kroatische Literatur einbezog. Und trotz des „Eisernen Vorhanges“ unternahmen die 
Landesbibliothekare gezielte Einkaufsfahrten in die Antiquariate von Budapest, Győr, Sopron, Székesfehérvár, Szombathely und Veszprém. Es hat sogar den Anschein, als hätte gerade die Existenz des „Eisernen Vorhanges“ das Bewusstsein um die Bedeutung des bibliothekarischen Fensters nach Osten bestärkt. Dadurch konnte mit der Zeit auch ein ansehnlicher „Altbestand“ aufgebaut werden, auf den die Bibliothek - es sei an das Gründungsjahr 1922 erinnert - nicht genuin zurückgreifen bzw. aufbauen konnte.

1970 erfolgte die Übersiedlung von Landesarchiv und -bibliothek vom „Leinner-Haus“ in einen eigens errichteten Zubau zum Gebäude der Burgenländischen Landesregierung. (Das Landesmuseum hatte bereits in den 1950ern ein eigenes Gebäude beziehen können.) In den darauffolgenden Jahren konnte sich die Landesbibliothek endgültig als wissenschaftliche Leitbibliothek des Burgenlandes - mit geschichtswissenschaftlichem Schwerpunkt - etablieren. Es bedarf keiner eigenen Erwähnung, dass all die Jahre hindurch sämtliches im Burgenland erscheinendes Schriftgut sowie sämtliche von burgenländischen Autoren verfassten oder herausgegebenen Publikationen Eingang in unsere Sammlung gefunden haben. Im Jahre 1980 konnte die Bibliothek die 70 000-Bände-Schwelle überschreiten. Die von der Bibliothek mitherausgegebenen Periodika (Burgenländische Heimatblätter, Burgenländische Forschungen) wurden mit 74 anderen Institutionen bzw. deren Publikationen ausgetauscht, wobei 21 davon in Ungarn situiert waren: Der Kalte Krieg konnte so eisig nicht sein, dass wir nicht Luft durch unser Fenster nach Osten ließen.

Dieses Fenster - ich weiß, dass ich soeben im Begriffe stehe, den Vergleich überzustrapazieren - erfuhr durch die nächste große Zäsur der Geschichte Europas, das Jahr 1989 und der Ereignisse in dessen Gefolge eine gravierende Erweiterung. Die Bibliothek nahm nun auch die Literaturen über die deutsche Wiedervereinigung sowie über das zerfallende Jugoslawien und dessen Nachfolgestaaten auf, ebenso über die anderen ehemals unter dem Begriff „Ostblock“ subsumierten Republiken. Eine zusätzliche Kursänderung unserer Sammelpolitik betraf die sogenannte „Belletristik“, deren Werke bislang ein deutlicher Haut- gout angehaftet und die man - abgesehen von den wenigen burgenländischen Eigenerzeugnissen auf diesem Gebiet - nur in Ausnahmefällen angeschafft hatte. Nun wurden auch Romane und Erzählungen angekauft, sofern sie sich den Regimewechseln, der Wiedervereinigung, den Stasidramen, Bürgerkriegen etc. widmeten und qualitativ als Quellen zur Rezeptionsgeschichte unserer jüngsten Vergangenheit gelten können.

Zuletzt noch ein Wort zu den ,wenigen belletristischen Eigenerzeugnissen des Burgenlandes“: Im Gegensatz zur großen Musik - man denke nur an Haydn und Liszt - ist auf dem Gebiet des heutigen Burgenlandes keine große Literatur gewachsen. Es gibt keine burgenländischen $\mathrm{Au}$ toren mit europäischer oder gar Weltgeltung und nur die wenigsten unserer Schreibenden bekamen zumindest einen Fuß in die Tür von Wien. Zurückzuführen ist das wohl auf die Tatsache, dass jahrhundertelang die Sprachen der Bildung hier Ungarisch und Latein waren, während das Deutsche, hierorts in weichen aber schweren Dialekten gesprochen, es als „Bauernsprache“ nicht zur Literaturfähigkeit geschafft hat. Nun ist das aber bald schon 100 Jahre her und scheint sich gerade eben zu ändern. Die Basis für diese Veränderung legten einerseits das Literaturhaus Mattersburg, andererseits der Verlag „Edition Lex Liszt 12“ in Oberwart, denen es gelungen ist, so etwas wie eine Literaturszene im Burgenland $\mathrm{zu}$ schaffen. Prompt scheint der Boden genug bereitet und es gibt junge Schreibende aus dem Burgenland, die zum Teil schon mit ersten Romanen österreichweit reüssieren konnten und zur Hoffnung berechtigen, die (derzeit) 140000 Bände unserer Landesbibliothek mit allerlei schönen und eigenwüchsigen Werken zu bereichern.

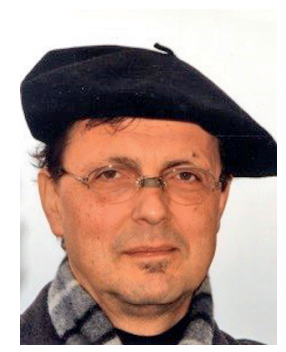

Dr. Jakob Michael Perschy

Referat Burgenländische Landesbibliothek Amt der Burgenländischen Landesregierung Landhaus, Europaplatz 1

A-7000 Eisenstadt

Österreich

Jakob.Perschy@bgld.gv.at 